\title{
4. PATTERN FORMATION IN HETEROGENEOUS DOMAINS
}

\author{
Debbie L. Benson, Philip K. Maini, and Jonathan A. Sherratt \\ Centre for Mathematical Biology \\ Mathematical Institute \\ 24-29 St. Giles' \\ Oxford OX1 3LB, UK
}

Development of spatial pattern in the early embryo results from the interaction of several processes in a complex hierarchy of mechanisms. Most models for morphogenesis to date have, however, focussed on a particular mechanism. Although such models are capable of capturing some aspects of development they are inconsistent with key experimental observations. Here we consider a two-step hierarchy of patterning mechanisms in which the spatial pattern of a control chemical regulates morphogen diffusivity in an overlying reaction diffusion system. Specifically, we consider the one-dimensional system

$$
\begin{aligned}
u_{t} & =\gamma f(u, v)+u_{x x} \\
v_{t} & =\gamma g(u, v)+\left(D(c) v_{x}\right)_{x} \\
c_{t} & =\nu c_{x x}-\theta c,
\end{aligned}
$$

where $u(x, t)$ and $v(x, t)$ are morphogen concentrations at position $x$ and time $t, c(x, t)$ is the concentration of control chemical, and $f$ and $g$ describe the chemical reaction kinetics. The system (4.1) has been nondimensionalised such that $u, v$ and $c$ have diffusion coefficients $1, D(c)$ and $\nu$ respectively, and we assume that $D=\alpha c$ where $\alpha$ is a positive constant; biologically, this could, for example, reflect an increase in gap junction permeability for $v$ due to the presence of $c$ (Othmer \& Pate 1980); $c$ is assumed to degrade with rate $\theta$, and $\gamma$ is a scale factor proportional to the length of the domain. The equations hold on the space domain $x \in[0,1]$ with zero flux boundary conditions on $u$ and $v$. The boundary conditions for $c$ are $c_{x}(0, t)=0, c(1, t)=c_{0}$. If we assume that the equation for $c$ reaches a stable equilibrium on a fast time scale during which insignificant changes in morphogen concentration take place, then $D=\alpha c_{0} \cosh (\delta x) / \cosh \delta$ where $\left.\delta=\sqrt{(} \theta / \nu\right)$. This represents a smoothly increasing diffusion coefficient for $v$ (cf. Figure 4.1(a)).

This system is mathematically intractable. However, approximating the diffusion coefficient of $v$ by the step function illustrated in Figure 4.1(b) does not change the qualitative form of the solutions of the original system, but enables us to carry out a linear analysis (Benson et al. 1993a) to delimit regions in parameter space wherein different types of pattern occur (cf. Figure 4.2). The crucial difference between the patterns illustrated in Figure 4.2 and those of reaction-diffusion models solved in a homogeneous environment is that, in the former case, pattern can be isolated in specific parts of the domain, in marked contrast to the case of homogeneous diffusivity. In the above analysis, the diffusion coefficient of $u$ was assumed constant across the domain. However, if we allow the diffusion coefficients of both morphogens to vary in a stepwise manner, the model exhibits patterns in which the wavelength can vary markedly across the domain (see Figure 4.3). We illustrate the application of the two 
(a)

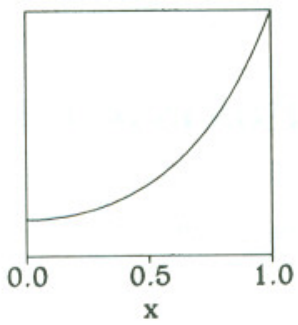

(b)

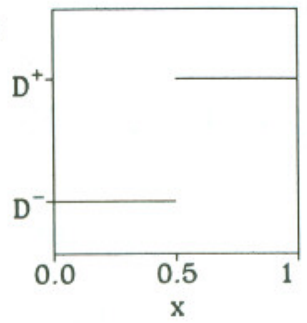

Figure 4.1. Different forms of diffusion coefficient $D$ used for system (4.1): (a) smoothly varying, (b) step function.
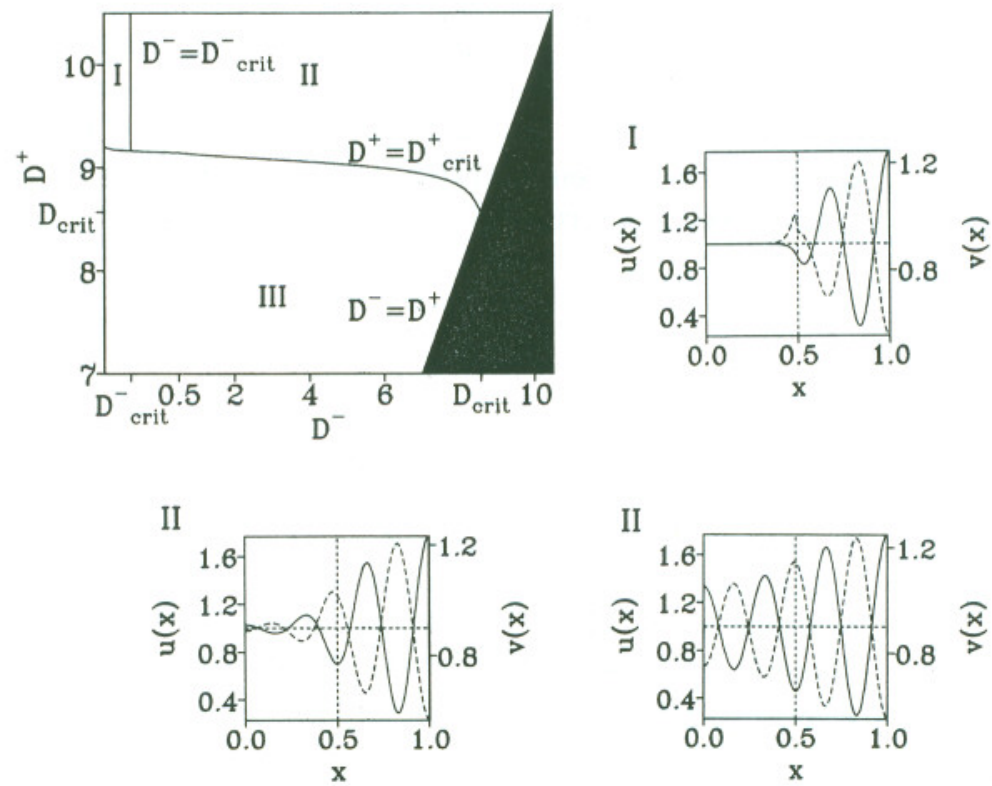

Figure 4.2. Diagram showing the three different regions (I,II,III) in parameter space wherein the three qualitatively different solution types arise. (Computations on the Schnackenberg (1979), reaction diffusion system: solution profiles $u-; v-\ldots)$. See Benson et al. (1993b) for parameter values.

step model by considering two experimental observations that contradict the predictions of the standard reaction-diffusion model, namely; the anterior-posterior asymmetry of skeletal elements in the limb (see, for example, Wolpert 1981); and the results of the experiments on double anterior composite limbs (Wolpert \& Hornbruch 1990) which show that one can produce limbs with two humeral elements while keeping the domain size constant.

The simulations illustrated by Figure 4.2 show that reaction diffusion systems with spatial variation in diffusion coefficients may produce isolated patterns and asymmetric oscillatory patterns. In our composite model, the underlying spatial pattern in control chemical influences the position and amplitude of peaks in morphogen concentration. If we apply this model to limb morphogenesis, the positional information (Wolpert 1981) supplied by these morphogen profiles could lead, via cell differentiation, to the specification of asymmetrically patterned elements, whose position within the domain can be controlled by the spatial distri- 

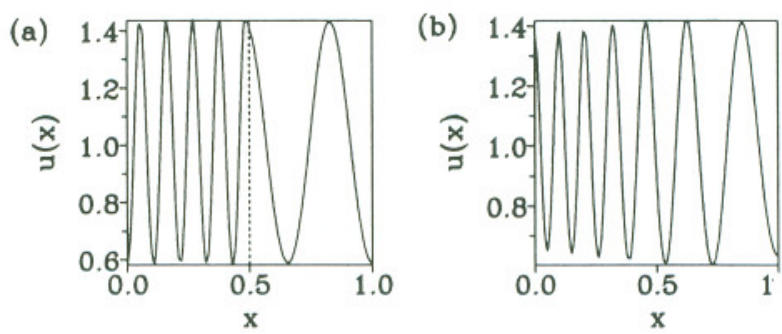

Figure 4.3. Solutions to (4.1) when the diffusion coefficients of both morphogens vary (a) stepwise, (b) continuously. Note that in (a) the wavelength varies discontinuously across the domain, but in (b) this variation is continuous. For parameter values, see Benson et al. (1993a). ( $u$ solution profile only shown $-v$ solution profile is approximately out of phase with that of $u$.)

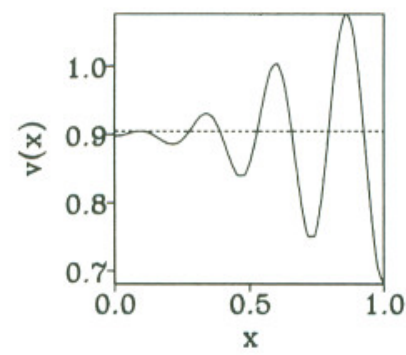

Figure 4.4. Steady state solution exhibited by (4.1) for a certain set of parameter values (see Maini et al. (1992)). For a suitably chosen threshold concentration, this prepattern specifies three skeletal elements which are intrinsically distinct because of the varying concentrations of morphogen to which they are each exposed. ( $u$ profile only shown).

bution of $c$. This is in contrast to the standard reaction-diffusion system with spatially uniform parameters, which produces identical and equally-spaced elements. The patterns produced by the model (4.1) are therefore more consistent with those observed in certain developmental processes, for example digit patterning in the vertebrate limb, than those exhibited by the standard model. This is illustrated in Figure 4.4 where, for a suitably chosen parameter set, the composite model exhibits a steady state solution with three peaks in concentration which, in contrast to the standard model, have different amplitudes of oscillation. A further consequence of the isolated solution profiles exhibited by the composite model is that duplication of skeletal elements can be predicted without necessarily increasing the length of the domain. For example, Figure 4.5 shows that imposing symmetric boundary conditions on the control chemical, $c$, but keeping the domain fixed in size, can lead to duplication. In this case, therefore, one can increase pattern complexity without it being necessary to increase the domain size. Hence, this is consistent with the results of (Wolpert \& Hornbruch 1990). Note that in the standard Turing model, one would predict, at least for low mode numbers, that the pattern complexity would remain unchanged for a fixed domain size (Dillon et al. 1992). 

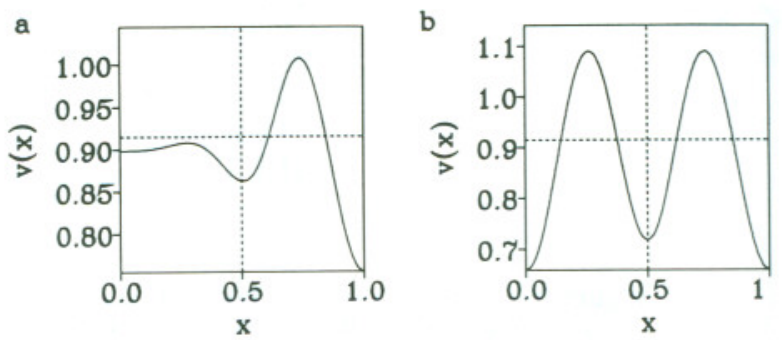

Figure 4.5. For the model (4.1), imposing symmetric boundary conditions on $c$ increases pattern complexity. (a) Solution for boundary conditions $c_{x}(0, t)=o, c(1, t)=c_{o}$, (b) solution for symmetric boundary conditions $c(0, t)=c(1, t)=c_{o}$. (u profiles only shown - see Maini et al. (1992) for parameter values).

ACKNOWLEDGMENTS DLB acknowledges the Wellcome Trust for a Prize Studentship in Mathematical Biology. JAS was supported by a Junior Research Fellowship at Merton College, Oxford.

\section{REFERENCES}

Benson, D. L., Maini, P. K., \& Sherratt, J. A. 1993a. Analysis of pattern formation in reaction diffusion models with spatially inhomogeneous diffusion coefficients. Math. \& Comp. Modelling. (To appear).

Benson, D. L., Sherratt, J. A., \& Maini, P. K. 1993b. Diffusion driven instability in an inhomogeneous domain. Bull. Math. Biol., 55, 365-384.

Dillon, R., Maini, P. K., \& Othmer, H. G. 1992. Pattern formation in generalized Turing systems. I. Steady-state patterns in systems with mixed boundary conditions. (To appear in J. Math. Biol.).

Maini, P. K., Benson, D. L., \& Sherratt, J. A. 1992. Diffusion models with spatially inhomogeneous diffusion coefficients. IMA J. Math. Appl. in Medic.\& Biol., 9, 197-213.

Othmer, H. G., \& Pate, E. 1980. Scale-invariance in reaction-diffusion models of spatial pattern formation. Proc. Natl. Acad. Sci. USA, 77, 4180-4184.

Schnackenberg, J. 1979. Simple chemical reaction systems with limit cycle behaviour. J. Theor. Biol., 81, 389-400.

Wolpert, L. 1981. Positional information and spatial patterning. Phil. Trans. R. Soc. Lond., B259, 441-450.

Wolpert, L., \& Hornbruch, A. 1990. Double anterior chick limb buds and models for cartilage rudiment specification. Development, 109, 961-966. 